\title{
BMJ Open A population-based observational study on the factors associated with the completion of palliative chemotherapy among patients with oesophagogastric
} cancer

\author{
Oliver Groene, ${ }^{1,2}$ Tom Crosby, ${ }^{3}$ Richard Henry Hardwick, ${ }^{4}$ Stuart Riley, ${ }^{5}$ \\ Kimberley Greenaway, ${ }^{6}$ David Cromwell ${ }^{1,2}$
}

To cite: Groene 0 , Crosby T, Hardwick RH, et al. A population-based observational study on the factors associated with the completion of palliative chemotherapy among patients with oesophagogastric cancer. BMJ Open 2015;5:e006724. doi:10.1136/bmjopen-2014006724

- Prepublication history and additional material is available. To view please visit the journal (http://dx.doi.org/ 10.1136/bmjopen-2014006724).

Received 24 September 2014 Revised 5 January 2015 Accepted 7 January 2015

CrossMark

For numbered affiliations see end of article.

Correspondence to Dr Oliver Groene; oliver.groene@|shtm.ac.uk

\section{ABSTRACT}

Objectives: Palliative chemotherapy is routinely given to patients diagnosed with locally advanced or metastatic oesophagogastric (0-G) cancer. We examine which patients with 0-G cancer in England receive palliative chemotherapy, and identify factors associated with treatment completion.

Design: A prospective population-based observational study.

Setting: All English National Health Service (NHS) trusts diagnosing patients with $0-\mathrm{G}$ cancer.

Participants: Data were prospectively collected on patients diagnosed with invasive epithelial cancer of the oesophagus or stomach between 1 October 2007 and 30 June 2009 in English NHS hospitals, and those who had palliative treatment intent.

Outcome measure: We calculated the proportion of patients with different characteristics (eg, age, sex, stage at diagnosis, performance status) starting palliative chemotherapy. Multiple logistic regression was used to identify characteristics associated with non-completion of chemotherapy.

Results: There were 9768 patients in the study whose treatment intent was palliative. Among these, 2313 (24\%) received palliative chemotherapy. It was received by $51 \%$ of patients aged under 55 years but only $9 \%$ of patients aged 75 years or over. Overall, 917 patients $(53 \%)$ completed their treatment among the 1741 patients for whom information on treatment completion was recorded. Treatment completion ranged from 50-60\% for patients with good performance status but was under $35 \%$ for patients aged 55 years or older with poor performance status. Treatment completion was not associated with site of cancer, pretreatment stage, sex, comorbidities or histology.

Conclusions: Completion rates of palliative chemotherapy in patients with $0-G$ cancer are low and elderly patients with poor performance status are very unlikely to complete a palliative chemotherapy treatment. Clinicians and patients should consider this information when balancing potential (survival) benefits, toxicity of treatment and its effect on quality of life.

\section{Strengths and limitations of this study}

- We examined completion of palliative chemotherapy among patients with oesophagogastric $(0-G)$ cancer.

- Of 9768 palliative patients with 0-G cancer in England, $2313(24 \%)$ received palliative chemotherapy.

- Treatment completion rate was $53 \%$, and was lower in older patients and patients with low performance status.

- Elderly patients with poor performance status are unlikely to complete palliative chemotherapy.

- Clinicians and patients need to balance survival benefits, quality of life and treatment toxicity.

\section{INTRODUCTION}

Treatment decision-making regarding palliative chemotherapy is complex. Patients and oncologists need to balance the potential benefit of increased survival against the risk of toxicity and its effect on quality of life. Over-optimistic assessments of treatment response and patients' ability to tolerate it could be associated with patients who start palliative chemotherapy and fail to complete the planned treatment, but there is little information on how often this occurs in practice.

This is of particular relevance in the context of oesophagogastric (O-G) cancer, a condition with poor prognosis and for which experimental evidence on palliative treatment is limited. O-G cancer is the fifth most common cancer and third most common cause of death from cancer in the UK. About 17000 patients are diagnosed every year in the UK and approximately 12500 deaths are registered per year. ${ }^{1}{ }^{2}$ Owing to the late onset of symptoms and diagnosis, only $35 \%$ 
of patients are suitable for treatment with curative intent and more than half of all patients die within the year of diagnosis. $^{3-5}$ To prolong survival and improve quality of life, palliative chemotherapy is given to patients with locally advanced or metastatic cancer. ${ }^{6} 7$

In the UK, O-G cancer therapy was influenced by the phase 3 randomised controlled trial (RCT) comparing epirubicin, cisplatin and infusion 5-fluorouracil (5-FU; ECF) with mitomycin, cisplatin and infusion 5-FU in patients with oesophageal or gastric tumours. ${ }^{8}$ This reported median survival times of 8-9 months for both arms, and ECF became the accepted standard. Recently, the REAL2 trial demonstrated epirubicin, oxaliplatin and capecitabine to be an alternative to ECF among patients with O-G cancer. ${ }^{9}$ However, for patients with metastatic oesophageal cancer, there is uncertainty about the median survival of patients on best supportive care alone, and a Cochrane review failed to demonstrate a survival benefit of palliative chemotherapy. ${ }^{10}$ The authors note that this "is in sharp contrast to the extended application of palliative chemotherapy in practice." ${ }^{10}$ The evidence of benefit is stronger for patients with gastric cancer. A recent meta-analysis found that palliative chemotherapy resulted in substantially improved survival compared with supportive care ( $\mathrm{HR}=0.37 ; 95 \%$ CI 0.24 to 0.55$)$, although its use needs to be balanced with the toxicity of the treatment. ${ }^{11}$

A limitation of the current evidence is the lack of outcome information for different patient groups. Various prognostic models for survival among patients receiving palliative chemotherapy have been developed and suggest that survival is worse for patients with poor performance status and presence of liver/peritoneal metastases. $^{12} 13$ However, these have not consistently included tumour site, histology, comorbidities, sex or age. This may reflect their development using trial data sets, and that patients of very old age or poor performance status are under-represented in clinical trials. ${ }^{14}$ Recent European Society for Medical Oncology (ESMO) guidelines for patients with gastric cancer recommend that organ function, performance status and comorbidities be considered, and that age alone is not a contraindication. ${ }^{15}$ The ESMO guideline for patients with oesophageal cancer recommended that palliative chemotherapy should be considered for patients with adenocarcinoma who have a good performance status. ${ }^{16}$

It is unknown how this evidence from controlled trials translates into routine clinical practice, and how studies on routine practice can help inform clinical decisionmaking. There has been a long-standing debate regarding the evidence generated by RCTs and observational studies, respectively. While RCTs are considered to be the gold standard to generate evidence for clinical decision-making, the contributions of observational studies can be significant, in particular in situations in which RCTs are unnecessary, inappropriate, impossible or inadequate. ${ }^{17}$ The average results of RCTs and well conducted observational studies may be remarkably similar and observational studies do not necessarily, as was previously assumed, overestimate the magnitude of treatment effects. ${ }^{18}{ }^{19}$ Moreover, observational studies are less prone to heterogeneity in results than RCTs due to the broader representation of the population at risk and the less restrictive inclusion and exclusion criteria of RCTs (age and comorbidities), making them more representative of clinical practice.

In this study, we contribute to this debate by addressing the routine clinical practice of palliative chemotherapy among patients with O-G cancer. This information, based on a national prospective cohort study, is of particular relevance to inform medical decision-making and patient choice as it is not subject to the strict exclusion criteria that are applied in RCTs. Specifically, we describe the characteristics of patients with O-G cancer who received palliative chemotherapy in England, report on the rate of treatment completion and identify patient factors associated with treatment completion.

\section{MATERIAL AND METHODS}

The National Oesophago-Gastric Cancer Audit (NOGCA) captured information on treatment patterns and outcomes on adult patients diagnosed with invasive epithelial cancer of the oesophagus or stomach between 1 October 2007 and 30 June 2009. It covered the process of diagnosis, staging and treatment planning as well as the surgical, oncological and endoscopic therapies delivered. Data on patients treated in English National Health Service (NHS) trusts (acute hospital organisations) were collected prospectively by hospital staff and submitted via a secure web-based tool to a central database. Details on rationale, data collection procedures and results of the audit have been reported elsewhere. $^{3} 4$

Data from the NOGCA are collected and analysed by the Royal College of Surgeons of England and the Health and Social Care Information Centre, supported through a grant from the Healthcare Quality Improvement Partnership. Permission for this analysis was granted by the NOGCA Clinical Reference Group. Data can be accessed through formal data accession request and approval by the Clinical Reference Group. The study was exempt from the UK National Research Ethics Committee approval as it involved analysis of data for service evaluation. Section 251 approval was obtained for the collection of the personal health data from the Ethics and Confidentiality Committee.

\section{Patient characteristics and study variables}

The study included all patients with a palliative treatment intent. Information was collected on patient characteristics such as age, sex, social deprivation index, tumour site, pretreatment stage, pretreatment histological diagnosis, performance status (Eastern Cooperative Oncology Group score), and comorbidities. $^{20} 21$ We grouped the site of cancer into two 
categories: oesophageal (oesophageal squamous cell carcinoma, upper or middle adenocarcinoma and lower or Siewert I adenocarcinoma) and gastric (gastrooesophageal junction Siewert II or III, and stomach). ${ }^{10} 11$ The audit collected information on the start of palliative chemotherapy, oncological treatment intent and whether patients completed the palliative chemotherapy regimen. The audit did not collect information on the regimen used.

The study captured treatment completion by adopting the data item from the National Cancer Dataset. ${ }^{22}$ The item captured whether treatment was delivered as planned or the reason for a change from this. Reasons for non-completion included: patient died, progressive disease during chemotherapy, acute toxicity, organisational problems and patient choice. This information was recorded by local clinicians and was calculated for first-line treatment only. As this is a national study, the number of participants was not calculated using a formal sample size calculation.

\section{Statistical analysis}

The study examined the proportion of patients with palliative treatment intent who received palliative chemotherapy, and the rate of treatment completion among patients receiving chemotherapy. These proportions were calculated for England overall, and for patients with various characteristics including age, sex, tumour site, pretreatment stage and performance status. The statistical significance of differences between individual characteristics were tested using $\chi^{2}$ tests.

We estimated the association between patient characteristics and the likelihood of treatment completion using multiple logistic regression with robust SEs. ${ }^{23}$ For two variables with missing data, we imputed values using multiple imputation by chained equation techniques by first creating multiple (10) copies of the data set and then fitting multiple logistic regression models to the imputed data sets. Variables in the imputation model included sex, age, site of cancer, diagnosis, pretreatment stage, performance status, comorbidities, deprivation and treatment completion. As recommended, imputed data on treatment completion was excluded from the regression model evaluating the association between patient characteristics and treatment completion. ${ }^{24}$

Finally, sensitivity analysis was performed to examine the effect of the data completion rate. First, we assessed how completion rates varied between NHS trusts with different levels of missing outcome data. Second, we examined two hypothetical scenarios in which (A) all patients with missing treatment outcome completed treatment and (B) none of the patients with missing treatment outcome completed treatment.

All analyses were performed in Stata V.11. All statistical tests are two-sided and $\mathrm{p}$ values lower than 0.05 were considered to show a significant result.
RESULTS

English NHS trusts submitted data on 16264 adult patients diagnosed with O-G cancer between 1 October 2007 and 30 June 2009 to the Audit. Of the 9768 patients with a palliative treatment intent, 2313 (23.7\%) underwent palliative chemotherapy (table 1 ). This treatment was more commonly used among younger patients and those with good performance status. Nonetheless, around $10 \%$ of patients aged 75 years or over, or who had a performance status of 2 or worse, received palliative chemotherapy. A lower proportion of women than men received palliative chemotherapy $\left(17.4 \%\right.$ vs $27.1 \% ; \chi^{2}$ test, $\mathrm{p}<0.001)$. Women were also less likely to undergo curative resection $(31.5 \%$ vs $36.5 \%, \mathrm{p}<0.001)$ and were more likely to be managed on a best-supportive-care pathway $(30.5 \%$ vs $23.9 \%, \mathrm{p}<0.001)$. However, the differences for the proportion of women receiving palliative chemotherapy become non-significant after adjusting for case-mix, due to the effect of age and performance status.

Information on treatment completion was available for $1741(75 \%)$ of the 2313 patients initiating chemotherapy. Among patients with known status of treatment completion, $917(52.7 \%)$ completed the chemotherapy, while 244 (14\%) died before all treatment was received. Treatment was stopped because of disease progression, toxicity of treatment or technical problems for 274 $(15.7 \%), 174(10 \%)$ and 3 patients, respectively. There were $129(7.4 \%)$ patients who chose to stop treatment.

Comparing the characteristics of patients with known and unknown treatment outcome, we found no significant differences with regard to sex, site of tumour, histology, pretreatment stage and performance status. A higher proportion of patients with unknown treatment outcome were missing information on pretreatment stage and performance status, and these patients had slightly fewer comorbidities recorded (online supplementary table S1).

The relationship between individual patient characteristics and the proportion of patients completing palliative chemotherapy is summarised in table 2. The rate of completion did not differ between men and women, or between oesophageal and gastric tumours, but the rate fell as the age of patients increased. Rates of completion among patients also decreased with lower performance status, number of comorbidities and the level of social deprivation.

Table 3 shows the results of the regression models for associations between patient characteristics and completion of chemotherapy. The first column presents the ORs for treatment completion in a data set in which information on all covariates was complete. The results are presented for each of the covariates individually, and then adjusted for all remaining variables reported in the table. After adjustment, patient age, performance status and level of deprivation remained independent predictors of the completion of chemotherapy. The second column presents the same data using the imputed data for all patients 
for whom the outcome (treatment completion) was known. Both data sets produced similar results.

The estimated rates of completion derived from the model suggested that the degree to which completion rates changed across the age groups varied among the different performance status scores (figure 1). However, a formal test for interaction between age and performance status proved to have insufficient statistical power to demonstrate this.

We further assessed the robustness of the estimates for treatment completion by conducting the two sensitivity analyses.

First, the overall treatment completion rate was not associated with the amount of missing data within NHS trusts: The rate was $49.2 \%$ for NHS trusts with no missing data $(95 \%$ CI $44.8 \%$ to $53.6 \%), 51.1 \%$ for trusts with $10-20 \%$ missing ( $47.5 \%$ to $54.7 \%$ ), $50.5 \%$ for trusts with
20-30\% missing $(45.4 \%$ to $55.6 \%)$ and $53 \%$ for trusts with more than $30 \%$ missing data ( $46.8 \%$ to $55.9 \%$ ).

Second, assuming that all patients with missing treatment outcome had completed their treatment gave an upper limit on the overall completion rate of $64.4 \%$. Assuming that none of the patients with missing treatment outcome had completed their treatment gave a lower limit of $39.6 \%$. We note that the true treatment completion rate is likely to be less than the $52.7 \%$ observed because patients for whom treatment completion was not available had worse performance status on average.

\section{DISCUSSION}

Main findings of the study

This study gives a national picture for the use of palliative chemotherapy among English patients with O-G

\section{Table 1 Patient selection for palliative chemotherapy among all patients with palliative treatment intent}

\begin{tabular}{|c|c|c|c|}
\hline \multirow[b]{2}{*}{ All patients } & \multirow{2}{*}{$\begin{array}{l}\text { Patients }(\%) \text { with palliative } \\
\text { treatment intent } \\
n=9768\end{array}$} & \multicolumn{2}{|c|}{$\begin{array}{l}\text { Patients (\%) undergoing } \\
\text { palliative chemotherapy }\end{array}$} \\
\hline & & $\mathrm{n}=2313$ & 23.7 \\
\hline \multicolumn{4}{|l|}{ Age } \\
\hline Under 55 & $670(6.9)$ & 344 & 51.3 \\
\hline $55-64$ & $1344(13.8)$ & 626 & 46.6 \\
\hline $65-74$ & $2437(24.9)$ & 853 & 35.0 \\
\hline 75 and over & 5317 (54.4) & 490 & 9.2 \\
\hline \multicolumn{4}{|l|}{ Gender } \\
\hline Female & 3429 (35.1) & 596 & 17.4 \\
\hline Male & 6339 (64.9) & 1717 & 27.1 \\
\hline \multicolumn{4}{|l|}{ Index of multiple deprivation } \\
\hline 1 (least) & $1806(18.5)$ & 502 & 27.8 \\
\hline 2 & $1832(18.8)$ & 471 & 25.7 \\
\hline 3 & 2016 (20.6) & 490 & 24.3 \\
\hline 4 & $1967(20.1)$ & 400 & 20.3 \\
\hline 5 (most) & $2147(22.0)$ & 450 & 21.0 \\
\hline \multicolumn{4}{|l|}{ Tumour } \\
\hline Oesophagus & $5686(58.2)$ & 1286 & 22.6 \\
\hline Stomach & $4082(41.8)$ & 1027 & 25.2 \\
\hline \multicolumn{4}{|l|}{ Diagnosis (histology) } \\
\hline Adenocarcinoma & 7411 (75.9) & 1884 & 25.4 \\
\hline Squamous cell & $1661(17.0)$ & 304 & 18.3 \\
\hline Other & $696(7.1)$ & 125 & 18.0 \\
\hline \multicolumn{4}{|l|}{ Pretreatment stage } \\
\hline 1 or 2 & $892(9.1)$ & 83 & 9.3 \\
\hline 3 & 1127 (11.5) & 239 & 21.2 \\
\hline 4 & 3896 (39.9) & 1377 & 35.3 \\
\hline Missing & $3853(39.5)$ & 614 & 15.9 \\
\hline \multicolumn{4}{|l|}{ ECOG/WHO performance status } \\
\hline 0 no restrictions & $1413(14.5)$ & 622 & 44.0 \\
\hline 1 restricted in strenuous activities & 1835 (18.8) & 620 & 33.8 \\
\hline 2 unable to work or worse & 3474 (35.6) & 326 & 9.4 \\
\hline Missing & 3046 (31.2) & 745 & 24.5 \\
\hline \multicolumn{4}{|l|}{ Comorbidities } \\
\hline None & $5317(54.4)$ & 1551 & 29.2 \\
\hline 1 & $2638(27.0)$ & 542 & 20.5 \\
\hline 2 or more & $1813(18.6)$ & 220 & 12.1 \\
\hline
\end{tabular}


cancer, and the proportion of patients who complete their prescribed therapy. Palliative chemotherapy was received by $23.7 \%$ of patients with palliative treatment intent, but was more commonly received by younger patients and those with good performance status. A substantially lower proportion of women than men received palliative chemotherapy; however, this can be partly explained by the effect of age (women were on average 5 years older at diagnosis) and other case-mix variables such as TNM classification stage (advanced disease). Residual differences may be explained by patient choice and preferences for a less aggressive approach to palliative care among women; however, data were not available to test these assumptions statistically.

The overall rate of treatment completion was $52.7 \%$. Information on the drug regimen for chemotherapy was not available for this study, but, assuming that the treatment selected was the most appropriate for the patient, it suggests that a substantial proportion of patients are not completing palliative chemotherapy, and raises questions about whether it is possible to improve the process of decision-making for patients and clinicians.

Higher completion rates were obtained in patients who were younger, had a good performance status and were from less deprived groups. Performance status is an established risk factor for predicting survival after palliative chemotherapy, and the ESMO guidelines for patients with oesophageal or gastric cancer recommend that patients undergoing treatment have good performance status. ${ }^{12} 131516$ Consistent with best practice guidelines, neither guideline recommends age as a factor to consider in treatment decisions, but our results suggest that age is associated with a lower completion of

Table 2 Relationship between patient characteristics and completion of chemotherapy

\begin{tabular}{|c|c|c|c|c|c|}
\hline & \multicolumn{2}{|c|}{$\begin{array}{l}\text { Patients (\%) with } \\
\text { known outcome* }\end{array}$} & \multicolumn{2}{|c|}{$\begin{array}{l}\text { Patients (\%) who } \\
\text { completed treatment } \dagger\end{array}$} & \multirow[b]{2}{*}{ p Value } \\
\hline & $\mathrm{n}=1741$ & 100 & $\mathrm{n}=917$ & 52.7 & \\
\hline \multicolumn{6}{|l|}{ Age } \\
\hline Under 55 & 268 & $(15.4)$ & 160 & 59.7 & \multirow{3}{*}{$<0.001$} \\
\hline $55-64$ & 479 & (27.5) & 273 & 57.0 & \\
\hline $65-74$ & 634 & (36.4) & 325 & 51.3 & \\
\hline Female & 454 & (26.1) & 244 & 53.7 & \multirow[t]{2}{*}{0.594} \\
\hline Male & 1287 & (73.9) & 673 & 52.3 & \\
\hline \multicolumn{6}{|l|}{ Index of multiple deprivation } \\
\hline 1 (least) & 393 & (22.6) & 229 & 58.3 & \multirow{2}{*}{0.008} \\
\hline 2 & 363 & (20.9) & 203 & 55.9 & \\
\hline \multicolumn{6}{|l|}{ Tumour } \\
\hline Oesophagus & 969 & $(55.7)$ & 505 & 52.1 & \multirow[t]{2}{*}{0.603} \\
\hline Stomach & 772 & (44.3) & 412 & 55.4 & \\
\hline \multicolumn{6}{|l|}{ Diagnosis } \\
\hline Adenocarcinoma & 1418 & (81.5) & 756 & 53.3 & \multirow{3}{*}{0.488} \\
\hline Squamous cell & 230 & (13.2) & 113 & 49.1 & \\
\hline Other & 93 & (5.3) & 48 & 51.6 & \\
\hline \multicolumn{6}{|l|}{ Pretreatment stage } \\
\hline 1 or 2 & 65 & ( 3.7$)$ & 37 & 56.9 & \multirow{4}{*}{0.822} \\
\hline 3 & 197 & (11.3) & 105 & 53.3 & \\
\hline 4 & 1088 & (62.5) & 576 & 52.9 & \\
\hline Missing & 391 & (22.5) & 199 & 50.9 & \\
\hline 2 or more & 182 & (10.4) & 82 & 45.1 & 0.045 \\
\hline
\end{tabular}


Table 3 Results of logistic regression models for associations between patient characteristics and completion of chemotherapy

\begin{tabular}{|c|c|c|c|}
\hline & \multicolumn{2}{|c|}{$\begin{array}{l}\text { Analysis of cases with complete data on } \\
\text { patient factors }(n=1291)\end{array}$} & \multirow{2}{*}{$\begin{array}{l}\text { Analysis using all patients } \\
\text { with known outcomes }(n=1741) \\
\text { Adjusted OR }(95 \% \mathrm{Cl})^{\star}\end{array}$} \\
\hline & Unadjusted OR & Adjusted OR* $(95 \% \mathrm{Cl})$ & \\
\hline \multicolumn{4}{|l|}{ Age } \\
\hline Under 55 & 1 & 1 & 1 \\
\hline $55-64$ & 0.89 & 0.89 (0.62 to 1.27$)$ & 0.90 (0.66 to 1.23$)$ \\
\hline $65-74$ & 0.71 & 0.75 (0.54 to 1.04$)$ & $0.75(0.55$ to 1.01$)$ \\
\hline 75 and over & 0.53 & $0.64(0.43$ to 0.97$)$ & 0.60 (0.43 to 0.84$)$ \\
\hline \multicolumn{4}{|l|}{ Gender } \\
\hline Female & 1 & 1 & 1 \\
\hline Male & 0.94 & $1.01(0.75$ to 1.37$)$ & $0.91(0.72$ to 1.14$)$ \\
\hline \multicolumn{4}{|l|}{ Index of multiple deprivation } \\
\hline 1 (least) & 1 & 1 & 1 \\
\hline 2 & 0.91 & 0.96 (0.64 to 1.43$)$ & $0.93(0.69$ to 1.25$)$ \\
\hline 3 & 0.75 & $0.71(0.46$ to 1.11$)$ & $0.78(0.58$ to 1.05$)$ \\
\hline 4 & 0.74 & $0.72(0.46$ to 1.13$)$ & 0.78 (0.57 to 1.07$)$ \\
\hline 5 (most) & 0.60 & 0.60 (0.40 to 0.90$)$ & 0.66 (0.49 to 0.91$)$ \\
\hline \multicolumn{4}{|l|}{ Tumour } \\
\hline Oesophagus & 1 & 1 & 1 \\
\hline Stomach & 1.05 & 1.08 (0.85 to 1.37$)$ & $1.10(0.89$ to 1.36$)$ \\
\hline \multicolumn{4}{|l|}{ Diagnosis } \\
\hline Adenocarcinoma & 1 & 1 & 1 \\
\hline Squamous cell & 0.85 & 0.85 (0.59 to 1.20$)$ & 0.81 (0.59 to 1.11$)$ \\
\hline Other & 0.93 & $0.96(0.54$ to 1.70$)$ & 0.97 (0.63 to 1.51$)$ \\
\hline \multicolumn{4}{|l|}{ Pretreatment stage } \\
\hline 1 or 2 & 1.17 & $1.58(0.58$ to 4.27$)$ & $1.27(0.67$ to 2.39$)$ \\
\hline 3 & 1.01 & 1.20 (0.52 to 2.74$)$ & 1.20 (0.83 to 1.73$)$ \\
\hline 4 & 1 & 1 & 1 \\
\hline \multicolumn{4}{|l|}{ ECOG/WHO performance status } \\
\hline 0 no restrictions & 1 & 1 & 1 \\
\hline 1 restricted in strenuous activities & 0.76 & $0.83(0.62$ to 1.10$)$ & 0.75 (0.58 to 0.96$)$ \\
\hline 2 unable to work or worse & 0.31 & $0.37(0.26$ to 0.53$)$ & $0.34(0.25$ to 0.46$)$ \\
\hline \multicolumn{4}{|l|}{ Comorbidities } \\
\hline None & 1 & 1 & 1 \\
\hline 1 & 0.87 & $0.94(0.71$ to 1.25$)$ & 0.94 (0.75 to 1.18$)$ \\
\hline 2 or more & 0.68 & $0.80(0.53$ to 1.20$)$ & 0.78 (0.56 to 1.09$)$ \\
\hline
\end{tabular}

treatment. ${ }^{25}$ Moreover, we observed that patients of older age are less likely to receive palliative chemotherapy. This clearly suggests that age is an important factor in decisions about treatment, although it is unclear whether this is driven by clinician or patient preferences, or both. Further research is required to understand these relationships, and how advice can be better tailored to patient age. Our finding that the completion rate of chemotherapy was not linked to tumour site, histology or sex is consistent with previous research. ${ }^{12-14}$

\section{Comparison with other studies and implications of results}

We were unable to identify other large prospective studies assessing completion of palliative chemotherapy for O-G cancer in actual practice. Data on toxicity of palliative chemotherapy treatment are usually collected alongside experimental studies. ${ }^{8}{ }^{26}$ However, the classification and grading of toxicity is not always systematic and, based on our data, only accounts for one-fifth of those who did not complete treatment. ${ }^{27}$ Moreover, experimental studies are often small and not powered to identify different factors associated with completion. ${ }^{10} 11$ They may also lack generalisability because patients of older age and lower performance status are typically under-represented. ${ }^{14}$

There has been some observational research on completion rates for other types of cancer, albeit not for palliative therapy. For example, US studies performed on the basis of the Surveillance, Epidemiology, and End Results (SEER) Medicare database suggest that completion rates of adjuvant chemotherapy in patients with epithelial ovarian cancer approximate $46.5 \%$; age and more than two comorbidities were identified as significant predictors. ${ }^{28}$ Using the same data source, the overall completion rate of adjuvant chemotherapy among patients with colon cancer was found to be $62.2 \%$, but this varied by age, marital status and level of comorbidity. ${ }^{29}$ While it is recognised that decisions regarding whether 


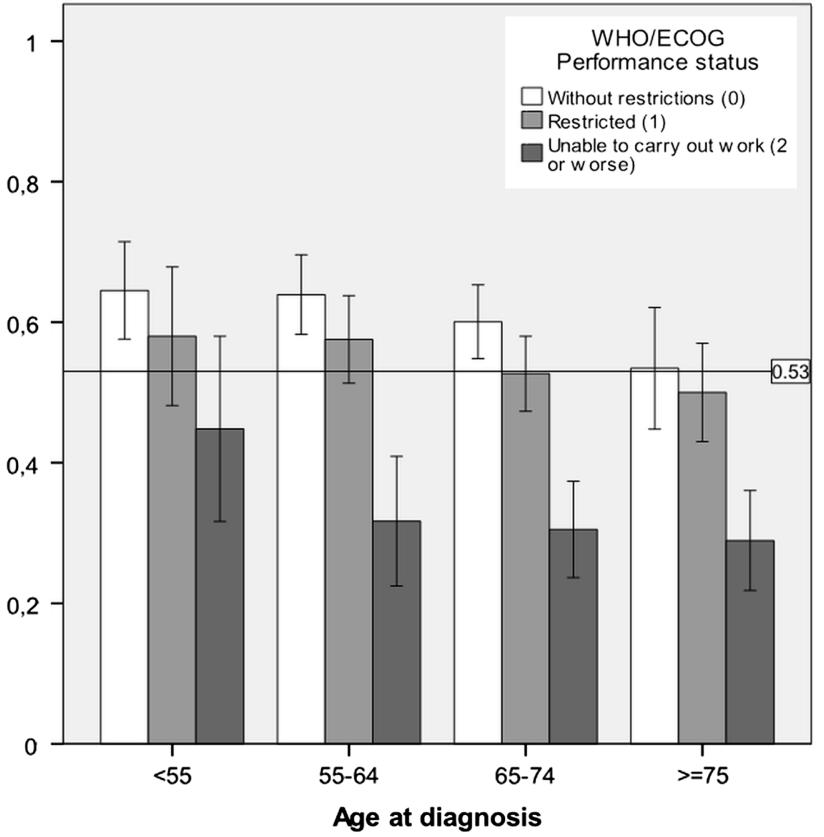

Figure 1 Completion of palliative chemotherapy, by age and performance status (adjusted for sex, deprivation, treatment stage, location of tumour and comorbidities). ECOG, Eastern Cooperative Oncology Group.

to continue treatment will be made differently in the curative and palliative setting, the factors associated with non-completion are similar to our study, suggesting that factors associated with appropriate patient selection for chemotherapy may also be similar across treatment settings.

Medical decision-making regarding palliative chemotherapy is complex and involves balancing clinical risk factors, potential benefits (including predicting survival) and harms, patient preferences and the probability of treatment completion. Palliative chemotherapy may prolong survival and quality of life 'on average', but this patient population will also contain people who respond and tolerate treatment poorly. While we do not have detailed quality of life analyses as part of this data set, it is highly likely that a substantial proportion of patients will not have benefited from treatment due to unacceptable toxicity, disease progression or death and that clinicians would not have chosen to treat these patients if these outcomes could have been predicted.

Enriching patient benefit populations is the fundamental concept behind personalised or stratified oncology. ${ }^{30}$ As well as finding biological factors or biomarkers that determine patient outcome and response to treatment, there is also a need to analyse factors associated with patient selection, such as age, performance status, extent of disease and patient choice. Lack of treatment completion may reflect an overly optimistic assessment of patients' likelihood to complete and benefit from treatment, or a preference of treatment over best supportive care among patients despite poor prognostic factors, or a lack of adequate institutional and social support services. ${ }^{31} 32$
Further studies should address the factors associated with treatment completion in more depth and assess the benefits of palliative chemotherapy not only in terms of survival but also in terms of health-related quality of life. The latter is a key issue as at present it is unclear how noncompletion of therapy is related to potential benefits of therapy. In other words, an incomplete palliative chemotherapy treatment stopped due to patient choice may still be associated with symptom relief and increased survival. However, non-completion may also be due to acute toxicity or death and in this case, given the short life expectancy of patients with O-G cancer on a palliative care pathway, may offer little benefit and in fact expose patients and their families to unnecessary distress. Research should also focus on whether treatment completion is associated with healthcare utilisation near end of life. Initiation of palliative chemotherapy in the last month of life is likely to reflect overtreatment of little benefit to patients.

\section{Study strengths and limitations}

The study was based on a large, prospective sample of patients diagnosed in 137 English NHS trusts, 89\% of all English NHS trusts providing O-G cancer care. The audit included $71 \%$ of all patients diagnosed in England during the audit period. ${ }^{3}$ This gives a comprehensive picture on how patients are managed in routine clinical service and not just the highly selected patient population that participates in clinical research. However, the study has a number of limitations. First, for some patients, information on performance status and pretreatment stage was missing. Assuming that data were "missing at random', we imputed missing data using chained equations. The estimates derived from this analysis are similar to those of the complete case analysis, suggesting that the model results are reliable. However, we cannot exclude the possibility of residual confounding because factors such as patients' psychological characteristics or social support networks can be important predictors of treatment completion. ${ }^{33}{ }^{34}$ A second limitation is that information on treatment completion was missing for a significant proportion of patients. The high proportion of missing data might be related to the administration of chemotherapy in the outpatient setting, natural disease progression and patient preferences, all of which contribute to the difficulty in assessing completion rates.

\section{CONCLUSION}

We assessed completion of treatment of patients receiving palliative chemotherapy for oesophageal and gastric cancer. We found that only $53 \%$ of patients completed their therapy. Key factors predicting non-completion are patients' age, performance status and level of deprivation. Elderly patients with incurable O-G cancer who have poor performance status are unlikely to complete palliative chemotherapy. Given the toxicity of the treatment, patients' likelihood to complete and benefit from 
treatment should be assessed carefully when deciding on treatment intent and modality.

\section{Author affiliations}

${ }^{1}$ Department of Health Services Research and Policy, London School of Hygiene \& Tropical Medicine, London, UK

${ }^{2}$ Clinical Effectiveness Unit, The Royal College of Surgeons of England, London, UK

${ }^{3}$ Velindre Cancer Center, Cardiff, UK

${ }^{4}$ Addenbrookes Hospital, Cambridge, UK

${ }^{5}$ Northern General Hospital, Sheffield, UK

${ }^{6} \mathrm{Health}$ and Social Care Information Centre, Leeds, UK

Contributors OG, TC, RHH, SR and DC designed the study, interpreted the results and drafted the manuscript. $O G$ and $K G$ were responsible for data acquisition and quality control. OG performed the statistical analysis. All authors edited the manuscript and approved the final version.

Funding The National Oesophago-Gastric Cancer Audit is commissioned by the Healthcare Quality Improvement Partnership (HQIP) as part of the National Clinical Audit Programme (NCA). HQIP is led by a consortium of the Academy of Medical Royal Colleges, the Royal College of Nursing and National Voices. Its aim is to promote quality improvement, and in particular to increase the impact that clinical audit has on healthcare quality in England and Wales. HQIP holds the contract to manage and develop the NCA Programme, comprising more than 30 clinical audits that cover care provided to people with a wide range of medical, surgical and mental health conditions. The programme is funded by NHS England, the Welsh Government and, with some individual audits, also funded by the Health Department of the Scottish Government, DHSSPS Northern Ireland and the Channel Islands.

Competing interests None.

Ethics approval Health Research Authority, Confidentiality Advisory Group.

Provenance and peer review Not commissioned; externally peer reviewed.

Data sharing statement No additional data are available.

Open Access This is an Open Access article distributed in accordance with the Creative Commons Attribution Non Commercial (CC BY-NC 4.0) license, which permits others to distribute, remix, adapt, build upon this work noncommercially, and license their derivative works on different terms, provided the original work is properly cited and the use is non-commercial. See: http:// creativecommons.org/licenses/by-nc/4.0/

\section{REFERENCES}

1. Cancer Research UK Statistical Information Team 2011: Cancer in the UK: April 2011. http://info.cancerresearchuk.org/cancerstats/ incidence/ (accessed 12 Jun 2014).

2. Cancer Research UK Statistical Information Team 2011: Common cancers-UK mortality statistics. http://info.cancerresearchuk.org/ cancerstats/mortality/cancerdeaths/ (accessed 12 Jun 2014).

3. Groene O, Cromwell D, Hardwick R, et al. National Oesophago-Gastric Cancer Audit. 2012 Annual Report. The Roya College of Surgeons of England, 2012. http://www.hscic.gov.uk/ catalogue/PUB06331/clin-audi-supp-prog-oeso-gast-2012-rep.pdf (accessed 19 May 2014).

4. Chadwick G, Groene O, Cromwell D, et al. National Oesophago-Gastric Cancer Audit. 2013 Annual Report. The Roya College of Surgeons of England, 2013. http://www.hscic.gov.uk/ catalogue/PUB11093/clin-audi-supp-prog-oeso-gast-2013-rep.pdf (accessed 19 May 2014).

5. Cancer Research UK Statistical Information Team 2011: Survival statistics for the most common cancers. http://info.cancerresearchuk. org/cancerstats/survival/latestrates/ (accessed 12 June 2014).

6. Scottish Intercollegiate Guidelines Network (SIGN). SIGN 87Management of oesophageal and gastric cancer. A national clinical guideline. Edinburgh: Scottish Intercollegiate Guideline Network (SIGN), 2006

7. Allum WH, Blazeby JM, Cunningham D, et al. Guidelines for the management of oesophageal and gastric cancer. Gut 2011;60:1449-72.

8. Ross $\mathrm{P}$, Nicolson $M$, Cunningham $D$, et al. Prospective randomized trial comparing mitomycin, cisplatin, and protracted venous-infusion fluorouracil (PVI 5-Fu) with epirubicin, cis5platin, and PVI 5-Fu in advanced esophagogastric cancer. J Clin Oncol 2002;20:1996-2004.

9. Cunningham D, Starlin N, Rao S, et al.; for the Upper Gastrointestinal Clinical Studies Group of the National Cancer Research Institute of the United Kingdom. Capecitabine and oxaliplatin for advanced esophagogastric cancer. N Engl J Med 2008;358:36-46.

10. Homs MYV, van der Gaast A, Siersema PD, et al. Chemotherapy for metastatic carcinoma of the esophagus and gastro-esophageal junction. Cochrane Database Syst Rev 2010;(5):CD004063.

11. Wagner AD, Unverzagt $S$, Grothe W, et al. Chemotherapy for advanced gastric cancer. Cochrane Database Syst Rev 2010;(3): CD004064.

12. Chau I, Norman AR, Cunningham D, et al. Multivariate prognostic factor analysis in locally advanced and metastatic esophago-gastric cancer-pooled analysis from three multicenter, randomized, controlled trials using individual patient data. J Clin Oncol 2004;22:2395-403.

13. Chau I, Ashley S, Cunningham D. Validation of the Royal Marsden hospital prognostic index in advanced esophagogastric cancer using individual patient data from the REAL 2 study. J Clin Oncol 2009;27: e3-4.

14. Saif MW, Makrilia N, Zalonis A, et al. Gastric cancer in the elderly: an overview. Eur J Surg Oncol 2010;36:709-17.

15. Okines A, Verheij M, Allum W, et al.; ESMO Guidelines Working Group. Gastric cancer: ESMO Clinical Practice Guidelines for diagnosis, treatment and follow-up. Ann Oncol 2010;21(Suppl 5): v50-4.

16. Stahl M, Budach W, Meyer JH, et al.; ESMO Guidelines Working Group. Esophageal cancer: clinical practice guidelines for diagnosis, treatment and follow-up. Ann Oncol 2010;21(Suppl 5):v46-9.

17. Black N. Why do we need observational studies to evaluate the effectiveness of health care. BMJ 1996;312:1215-18

18. Benson K, Hartz AJ. A comparison of observational studies and randomized, controlled trials. N Engl J Med 2000;342:1878-86.

19. Concato J, Shah N, Horwitz RI. Randomized, controlled trials, observational studies, and the hierarchy of research designs. $N E n g$ J Med 2000;342:1887-92.

20. Office of the Deputy Prime Minister. The English Indices of Deprivation 2004: summary (revised) http://www.communities.gov.uk/documents/ communities/pdf/131206.pdf, Neighbourhood renewal unit, 2004.

21. Oken MM, Creech RH, Tormey DC, et al. Toxicity and Response Criteria of the Eastern Cooperative Oncology Group. Am J Clin Oncol 1982;5:649-55.

22. The NHS Information Centre. Cancer dataset project. Cancer data manual. Version 4.5, Leeds: The NHS Information Centre, 2006.

23. Tan A, Freeman JL, Freeman DH. Evaluating health care performance: strengths and limitations of multilevel analysis. Biometrica J 2007;49:707-18.

24. Royston P. Multiple imputation of missing values: further update of ice, with an emphasis on categorical variables. Stata J 2009;9:466-77.

25. MacMillan Cancer Support. Cancer services coming of age: learning from the improving cancer treatment assessment and support for older people project. MacMillan Cancer Support, Department of Health and Age UK, 2012.

26. Tebbutt NC, Norman A, Cunningham D, et al. A multicentre, randomised phase III trial comparing protracted venous infusion (PVI) 5-fluorouracil (5-FU) with PVI 5-FU plus mitomycin C in patients with inoperable oesophago-gastric cancer. Ann Oncol 2002;13:1569-75

27. MacDonald JS, Haller D, Mayer RJ. Grading of toxicity. Manual of oncologic therapeutics. Philadelphia, PA: Lippincott, 1995:519-23.

28. Fairfield KM, Murray K, Lucas FL, et al. Completion of adjuvant chemotherapy and use of health services for older women with epithelial ovarian cancer. J Clin Oncol 2011;29:3921-6.

29. Hu CY, Delclos GL, Chan W, et al. Assessing the initiation and completion of adjuvant chemotherapy in a large nationwide and population-based cohort of elderly patients with stage-III colon cancer. Med Oncol 2011;28:1062-74.

30. Curtis C, Shah SP, Suet-Feung C, et al. The genomic and transcriptomic architecture of 2,000 breast tumours reveals nove subgroups. Nature 2012;486:346-52.

31. Koedoot CG, de Haan RJ, Stiggelbout AM, et al. Palliative chemotherapy or best supportive care? A prospective study explaining patients' treatment preference and choice. Br J Cancer 2003;89:2219-26.

32. Gott M, Ingleton $\mathrm{C}$, Bennett MI, et al. Transitions to palliative care in acute hospitals in England: qualitative study. BMJ 2011;342:d1773.

33. Braga S. Why do our patients get chemotherapy until the end of life? Ann Oncol 2011;22:2345-8.

34. Näppä U, Lindqvist $\mathrm{O}$, Rasmussen $\mathrm{BH}$, et al. Palliative chemotherapy during the last month of life. Ann Oncol 2011;22:2375-80. 